\title{
Helical Graphene Nanostructures Embedded with an Azulene Cluster
}

Naoki Ogawa, Yousuke Yamaoka, Hiroshi Takikawa, Ken-ichi Yamada,\# and Kiyosei Takasu*

${ }^{1}$ Graduate School of Pharmaceutical Sciences, Kyoto University, Yoshida, Sakyo-ku, Kyoto 606-

8501, Japan

${ }^{\#}$ Current address: Graduate School of Pharmaceutical Sciences, Tokushima University, Shomachi,

Tokushima 770-8505, Japan

*e-mail: kay-t@pharm.kyoto-u.ac.jp

\begin{abstract}
An azulene cluster is a structural defect of graphenes, and it alters the electronic, magnetic, and

structural properties of graphenes and graphene nanoribbons. However, detailed experimental

investigations of graphenes or graphene nanostructures embedded with an azulene cluster are limited

because they are difficult to synthesise. Herein, azulene-embedded graphene nanostructures (AGNs)

were synthesised by following a newly developed synthetic protocol. The nanostructures comprising

pentagons, hexagons, and heptagons has three characteristic edges, viz., zigzag-, armchair-, and

cove-type edges. Experimental and theoretical investigations of the properties of AGN revealed that
\end{abstract}


the cove edge has stable helical chirality with a racemisation barrier of $29.2 \mathrm{kcal} / \mathrm{mol}$, in contrast to normal cove-type edges that undergo rapid racemisation. The in-solution self-association behaviour and the structural, electronic, and electrochemical properties of AGN are also described in detail.

\section{Graphical Abstract}

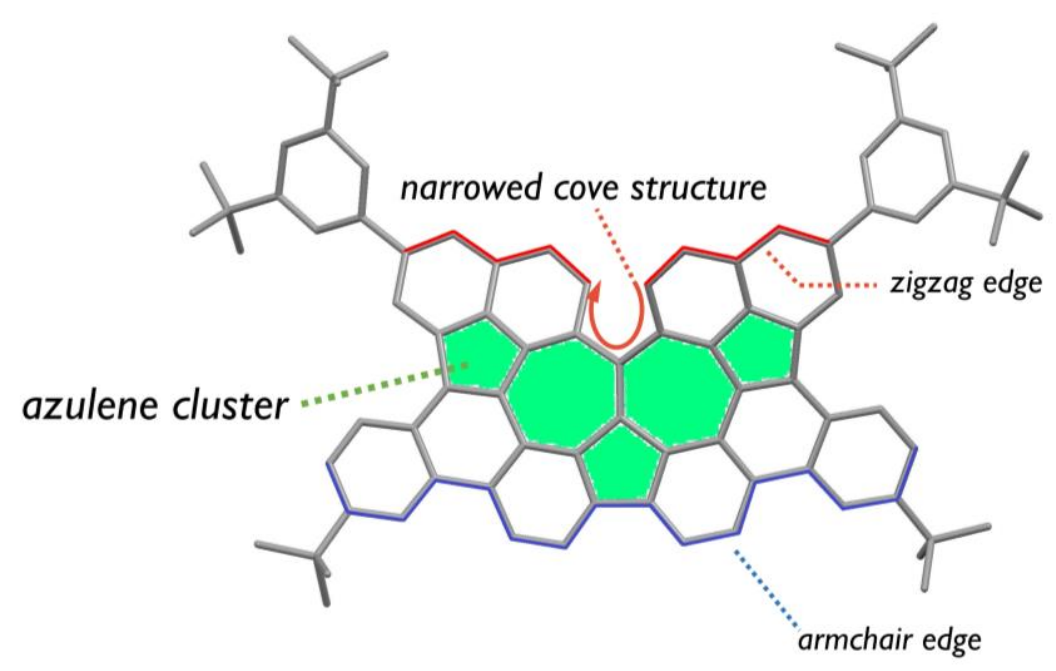


The topology of $\pi$-electrons is one of the most crucial factors that determine the properties of graphene nanostructures. Accordingly, much effort has been devoted towards synthesising and elucidating the properties of topologically diverse graphene nanostructures, whose hexagonal rings are fused in different patterns. ${ }^{1-4}$ Researchers have also been interested in introducing non-hexagonal rings such as pentagons, ${ }^{5-7}$ heptagons, ${ }^{8-10}$ or octagons ${ }^{11}$ into $\pi$-conjugation systems to achieve unique optical, electronic, and structural properties, distinct from those of $\pi$-systems composed of only hexagonal units.

Among the non-hexagonal ring systems, the contiguous pentagon-heptagon ring system, the socalled azulene structure, has been attracting considerable interest owing to its interesting properties such as a low-lying excited state and high dipolar moment, which are distinct from those of usual benzenoids. ${ }^{12}$ Thus, considerable efforts have been dedicated to incorporate azulene structures into polycyclic aromatic hydrocarbons (PAHs) to impart unique structural and electronic properties. ${ }^{13-17}$ Of particular interest is the azulene structure observed in graphenes as one of the structural defects, which breaks the symmetry of graphenes to significantly alter their mechanical, magnetic, and electronic properties, as well as open non-zero band gap. ${ }^{18-21}$ It is noteworthy that the azulene units in a single graphene molecule not only exist separately, rather tend to accumulate to form clusters. ${ }^{21}$ In graphene nanoribbons (GNRs), whose edge structures are important for their properties, the incorporation of azulene units results in unique edge structures. For example, the GNR bearing 
sandwiched contiguous azulene rings would have a zigzag edge and an armchair at the top and the bottom sides, respectively (Fig 1a). A "narrowed" variant of the cove-type edge ${ }^{22-26}$ (Fig. 1c) can also be considered as another example, where the "cove" comprises a contiguous 6-7-7-6 ring system instead of the four hexagons of a normal cove edge (Fig. 1b), leading to a narrower cove structure.

a

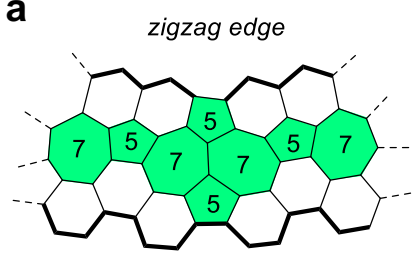

b

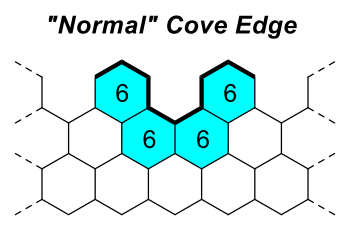

C

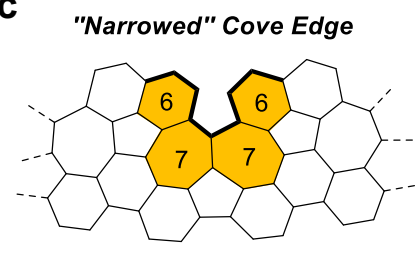

armchair edge

d

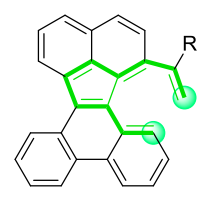

2

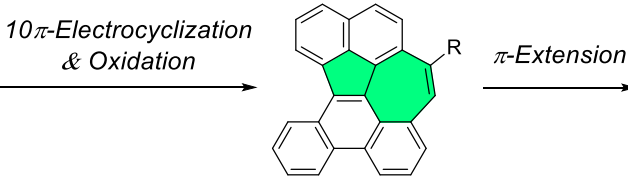

3

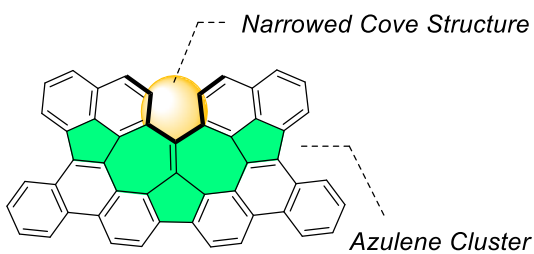

1

Fig. 1: Azulene-emmbedded graphenes and graphene nanostructures. a, One example of a

graphene nanoribbon containing clusters of azulene units. b, Structures of "normal" cove edge. c,

"Narrowed" variant of cove edge. d, Structural design and synthetic strategy of nanographene $\mathbf{1}$

While there were considerable efforts to theoretically elucidate the nature of azulene-embedded

graphenes and GNRs, ${ }^{18-21}$ detailed experimental investigations are still limited, probably due to their poor availability. Although several approaches based on chemical vapour deposition (CVD) 
techniques are emerging, a precise synthesis of the structurally uniform graphenes with azulene units is not straightforward since isomerisation among 5-, 6-, and 7-membered rings occurs on metal surfaces at elevated temperatures under typical conditions of the CVD process. ${ }^{27,28}$ Bottom-up chemical synthesis of nanocarbons that resemble model structures of such graphenes would be one solution to this problem. ${ }^{29-31}$ Since direct investigation of graphenes embedded with azulene units is challenging due to the difficulty in synthesis, it is of significance to prepare model nanocarbons to gain insights into the properties of parent graphenes. Compared to those of other nanocarbons composed only of hexagonal rings and isolated non-hexagonal ring(s), ${ }^{29-31}$ the bottom-up chemical syntheses of nanocarbons with multiple azulene units are still limited, because of the absence of reliable methods, especially in-solution ones. ${ }^{32-34}$ Peña, Pascual and co-workers, ${ }^{35}$ and Müllen, Fasel, Feng and co-workers ${ }^{36}$ elegantly addressed this situation by utilising on-surface dehydrocyclisation of rationally designed precursors to incorporate azulene units into nanocarbons. Mastalerz $^{37}$ and Zhang ${ }^{38}$ also independently achieved in-solution synthesis of highly fused azulene units via the Scholl cyclisation of ingeniously tailored precursors. Despite these pioneering works, there is still a lack of appropriate methods to construct highly condensed azulene clusters, structures that frequently appear in graphenes. ${ }^{21}$ Therefore, new synthetic protocols should be developed to access nanocarbons embedded with this moiety.

Herein, we report a bottom-up chemical synthesis of azulene-embedded graphene nanostructures 
1 and their properties (Fig. 1d). Compounds 1 have unprecedented contiguous azulene structures

condensed at their heptagonal rings, and it was designed as a model structure of one of the clustering azulene units observed in graphenes. It also has a helical structure considered to be a minimum structure of one of the narrowed variants of the cove-type edge. A newly developed $10 \pi-$ electrolysation-oxidation cascade of fluoranthene $\mathbf{2}$ to construct an azulene structure enabled us to obtain the key intermediate $\mathbf{3}$, leading to the successful generation of a highly condensed azulene cluster via further $\pi$-extension.

\section{Results and discussion}

In our continuing studies on PAHs, ${ }^{39}$ we reasoned that constructing an additional heptagonal ring next to the pentagon of benzo-fused fluoranthene $\mathbf{2}$ would be a straightforward way to obtain condensed azulene structure 3 , because 2 itself has a pentagonal ring surrounded by multiple benzene rings (Fig.1d). Unfortunately, it was found that conventional $\pi$-extension reactions such as metal-catalysed cycloisomerisation of alkyne, intramolecular Friedel-Crafts acylation, and Scholl cyclisation are not applicable for this purpose, as these reactions led to the formation of complex mixtures or recovery of unreacted starting materials (see Supporting Information for details). After several investigations, it was established that compound $\mathbf{2 c}$ consisting of an $\alpha$-styryl moiety is a suitable precursor to generate the desired azulene unit. Thus, activating this molecule by visible- 
light-irradiation in the presence of an oxidant resulted in a clean conversion of $\mathbf{2} \mathbf{c}$ into $\mathbf{3 c}$ via an oxidative cyclisation process (Fig. 2a, Eq. 1). The utilisation of visible light as a mild energy source was important to effectively generate the azulene structure since the activation of $\mathbf{2 c}$ by both UV light (low-pressure mercury-vapour lamp), which is conventionally used to promote $6 \pi$ electrocyclisation of stilbenes, ${ }^{40}$ and heating to $250{ }^{\circ} \mathrm{C}$ led to lower yields of $\mathbf{3 c}$ ( 52 or $40 \%$ yield, respectively). Since $\mathbf{2 c}$ has a $10 \pi$-electron system, this oxidative cyclisation reaction would proceed via a visible-light-promoted $10 \pi$-electrocyclisation ${ }^{41,42}$ followed by a dehydrogenation by the oxidant. Such an approach for azulene rings based on oxidative electrocyclic ring closure of the heptagon is an unprecedented one, to the best of our knowledge. The synthetic utility of this newly developed method was highlighted by applying it in the synthesis of boronate ester 3a from 2a (Fig. 2a, Eq. 2), as the C-B bond on its heptagon was converted into $\mathrm{C}_{\mathrm{sp}^{2}}-\mathrm{C}_{\mathrm{sp}^{2}}$ bonds by a transition-metal catalysed cross-coupling reaction such as the Suzuki coupling reaction, offering opportunities for further $\pi$-extensions. ${ }^{43}$ 
a

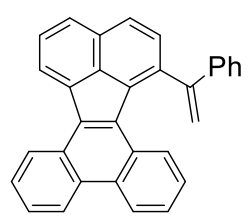

2c

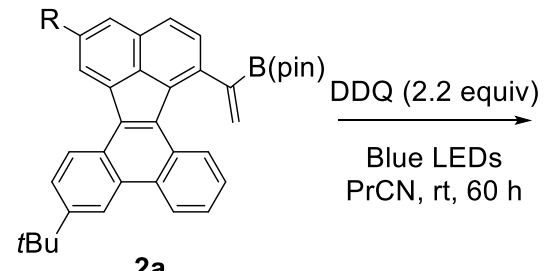

$\left(\mathrm{R}=3,5-(\mathrm{tBu})_{2} \mathrm{C}_{6} \mathrm{H}_{3}\right)$

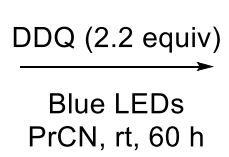

$\mathrm{PrCN}, \mathrm{rt}, 60 \mathrm{~h}$
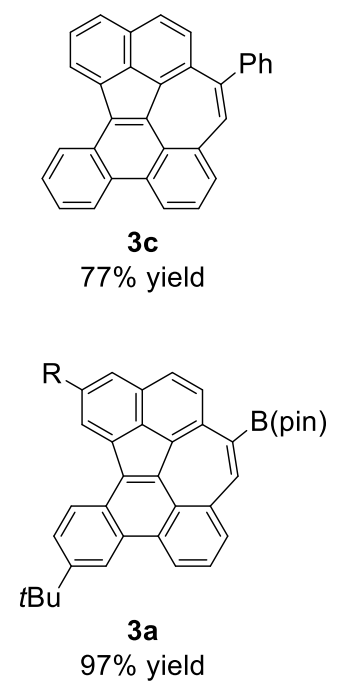

(1)

b

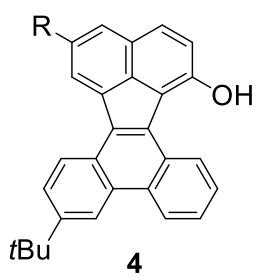

(a)

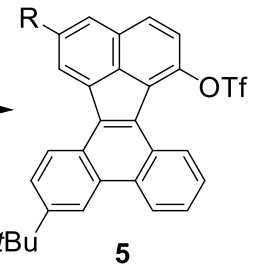

(b)

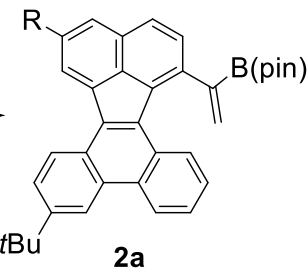

(c)<smiles></smiles>

(d)

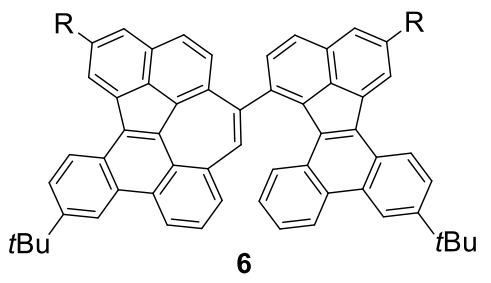

(e)

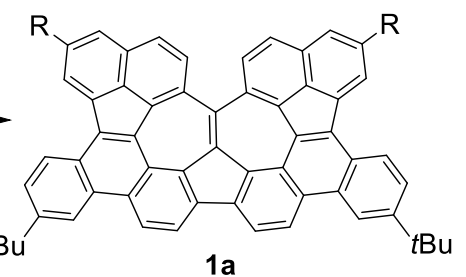<smiles></smiles>

Fig. 2: Synthetic protocol for the azulene units. a, Conversion of dibenzofluoranthene 2 into azulene 3 via visible-light-promoted oxidative cyclisation. b, Synthesis of AGN 1a. Reaction conditions: (a) $\mathrm{Tf}_{2} \mathrm{O}$, DIPEA, DCM, $25^{\circ} \mathrm{C}$ (b) $\mathrm{CH}_{2}=\mathrm{C}(\text { Bpin })_{2}, \mathrm{~K}_{3} \mathrm{PO}_{4}, \mathrm{Pd}\left(\mathrm{PPh}_{3}\right)_{4}$, dioxane, $100{ }^{\circ} \mathrm{C}$,

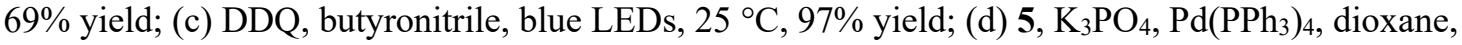


$100{ }^{\circ} \mathrm{C}, 46 \%$ yield; (e) DDQ, TfOH, DCM, $25^{\circ} \mathrm{C}, 5 \mathrm{~min}, 16 \%$ yield. $\mathrm{Tf}=$ trifluoromethanesulfonyl; DIPEA = diisopropylethylamine; DCM = dichloromethane; pin = pinacolate; DDQ = 2,3-dichloro5,6-dicyano- $p$-benzoquinone; LED = light emitting diode.

With azulene-embedded boronate ester 3a in hand, we moved forward to the synthesis of graphene nanostructure $\mathbf{1}$ (Fig. 2b). The cross-coupling reaction between boronate ester 3a and triflate $\mathbf{5}$, which was prepared by the triflylation of hydroxyfluoranthene $4,{ }^{39}$ proceeded smoothly to generate compound $\mathbf{6}$ in $46 \%$ yield. The Scholl reaction of compound $\mathbf{6}$ using the combination of 2,3-dichloro-5,6-dicyano- $p$-benzoquinone (DDQ) and trifluoromethanesulfonic acid (TfOH) resulted in the formation of two $\mathrm{C}-\mathrm{C}$ bonds to afford the desired graphene nanostructure 1a in $16 \%$ yield. The purple crystals of compound 1a suitable for X-ray crystallography were obtained by recrystallising it from a $\mathrm{NO}_{2} \mathrm{Ph} / \mathrm{CS}_{2} /$ cyclohexane solvent system by slow solvent evaporation, and its structure was unambiguously confirmed (Fig. 3a). ${ }^{44}$ The bond lengths of the pentagonal and heptagonal rings in compound 1a are summarised in Fig. $3 \mathrm{~b}$ together with those of the unsubstituted azulene. ${ }^{45}$ The azulene structures in compound 1a show significant bond alternation varying between $1.33 \AA$ and $1.50 \AA$, as observed in the unsubstituted azulene. The armchair-edge-like side of 1a, shown as the bottom side in the figure, has a rather planar structure, and 1a was stacked together on that side in the crystal form (Fig. $3 \mathrm{c}$ and $3 \mathrm{~d}$ ). The cove region of compound 1a has, in contrast, a 
significantly distorted helical structure, and the angle of the eclipsed rings (shown as B and B' in

Fig. $5 \mathrm{a})$ is $39^{\circ}$, the value is close to that reported for the normal cove edge $\left(38^{\circ}\right) .{ }^{22}$ The enantiomers arising from this cove region were alternatively arranged in the crystal structure with a distance as short as $3.2 \AA$, suggesting strong intermolecular $\pi-\pi$ stacking interactions (Fig. $3 \mathrm{c}$ and $3 \mathrm{~d}$ ).

a

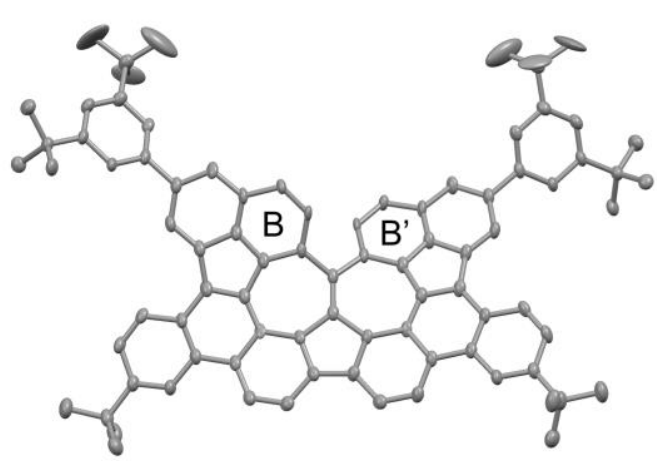

b

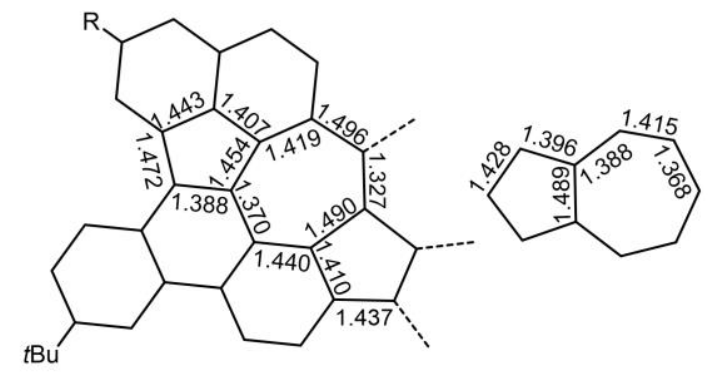

c
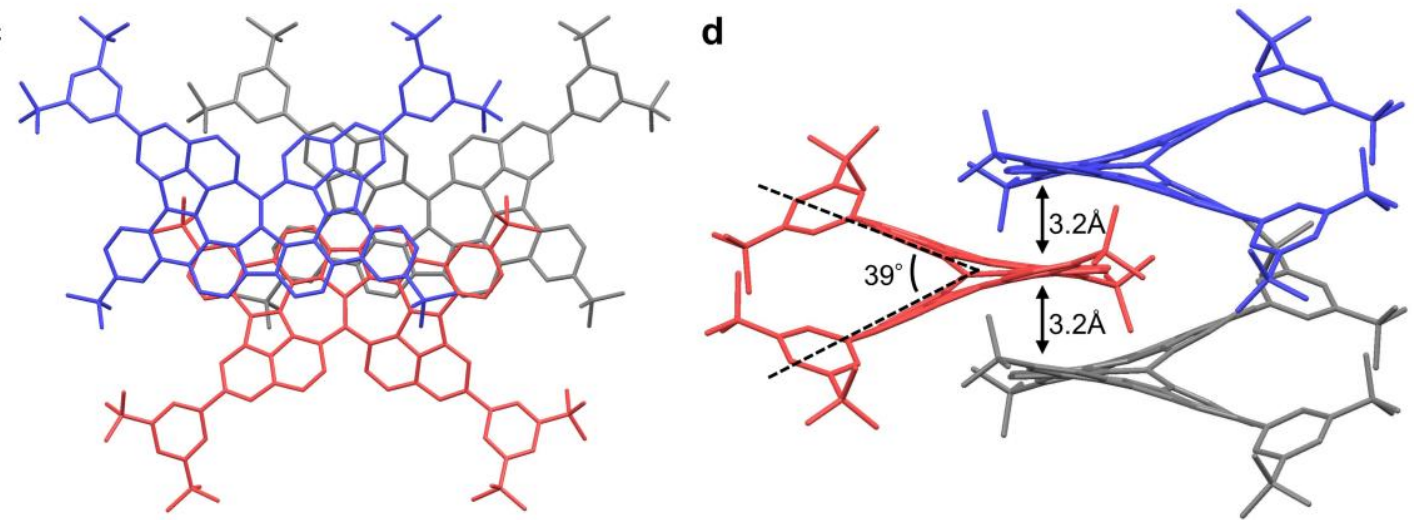

Fig. 3: Crystal structure of 1a. Hydrogen atoms and solvents are omitted for clarity. a, Top

view (thermal ellipsoids are shown at 50\% probability). b, Bond length of compound 1a (left) and azulene (right). c, Top view and, $\mathbf{d}$, side view of the packing structure in the crystal. 
The strong intermolecular interactions of 1a also led to an in-solution self-association behaviour: the ${ }^{1} \mathrm{H}$ NMR spectra of compound 1a showed a significant concentration-dependence; that is, the peaks of aromatic protons shifted upfield as the concentration increased. These upfield-shifts resulted from face-to-face aggregations, ${ }^{46}$ and the association constant $\left(K_{2}\right)$ in $\mathrm{CDCl}_{3} / \mathrm{CS}_{2}(1: 1)$ was estimated to be $74.1 \pm 25.5 \mathrm{M}^{-1}$ by assuming that dimers are formed predominantly $\mathrm{y}^{46,47}$ (see the Supporting Information for details). Interestingly, the degree of concentration dependence of the chemical shift varied significantly with the position of protons: the chemical shifts of the protons located on the planar region were considerably affected by the concentration, whereas those of the protons located around the cove region was not. These observations indicated that the in-solution self-aggregation was induced by the face-to-face $\pi-\pi$ stacking interactions between the planar regions.

Since the cove-type structure of 1a comprising of a contiguous 6-7-7-6 ring system was not investigated previously, we questioned if the racemisation barrier of this cove is high enough for 1a to be chiral. Since the direct separation of compound 1a by chiral HPLC was challenging due to its poor solubility, compound $\mathbf{1 b}$, whose aromatic substituent was replaced by a bulky tertiary alkyl side chain to increase its solubility, was prepared in a similar way. Compound $\mathbf{1 b}$ exhibited a significantly improved solubility in common non-polar organic solvents (even in hexane), but not in polar solvents such as $\mathrm{MeOH}$. Accordingly, $\mathbf{1 b}$ was subjected to chiral HPLC analyses, and the 
enantiomers were separated without any observable racemisation at $25^{\circ} \mathrm{C}$. The mirror-symmetric circular dichroism (CD) spectra of the two separated fractions confirmed that they are certainly a pair of enantiomers (Fig. 4a). The stable helicity of the cove of $\mathbf{1}$ is in clear contrast to that of the normal cove structure comprising of four benzene rings, or [4] helicene structure, which is reported to racemise immediately. ${ }^{22,48}$ Theoretical calculations revealed that the free energy of activation for the racemisation of the model structure (1c) is as high as $29.2 \mathrm{kcal} / \mathrm{mol}$ (Fig. 4b), which lies between those of [5]helicene and [6] helicene (24.6 and $36.2 \mathrm{kcal} / \mathrm{mol}$, respectively) (Fig. 4c). ${ }^{49}$ The reason for such a high racemisation barrier is that the hydrogen atoms of the cove region are forced to locate closely to each other owing to the large interior angle of the 7-membered ring, requiring a highly distorted achiral transition state to undergo racemisation. 
a

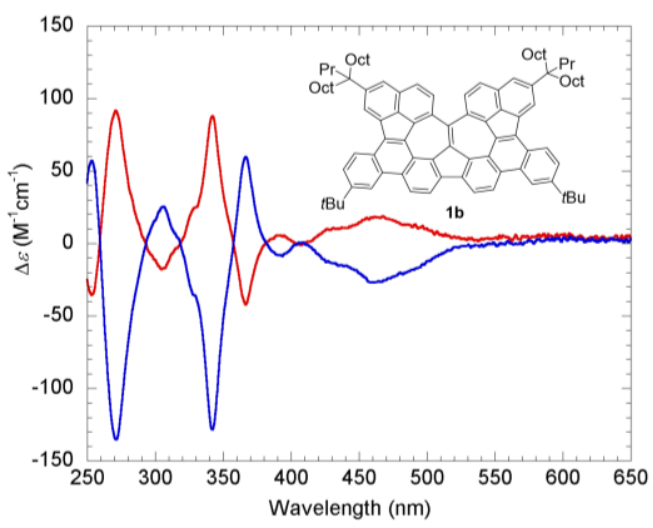

b

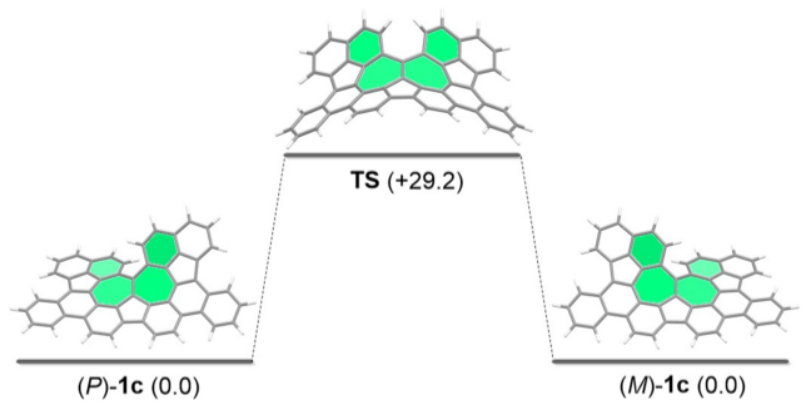

C

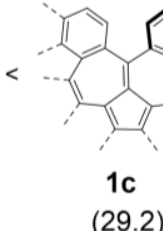

(24.6) d

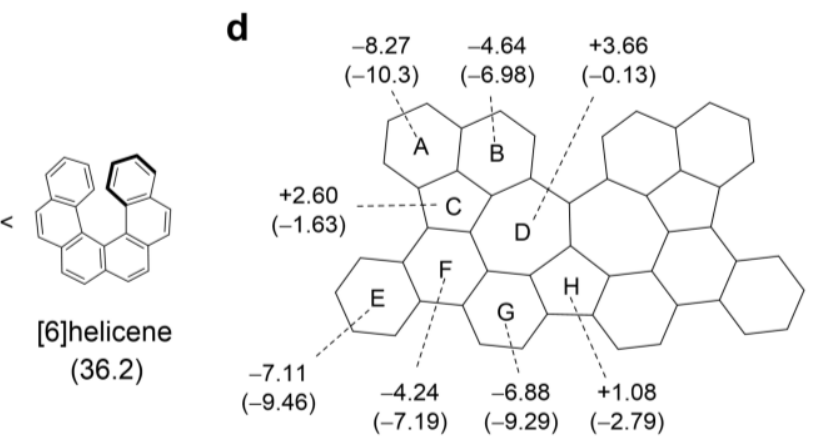

[6]helicene

(36.2)

Fig. 4: Chirality and local aromaticity of AGN 1. a, CD spectra of $1 \mathbf{b}\left(5.0 \times 10^{-5} \mathrm{M}\right.$ in hexane). b,

Process for the racemisation of the model compound 1c. Relative Gibbs free energies (kcal/mol) are given in parentheses. Calculation was carried out at the B3LYP/6-31G(d,p) level of theory. c, Comparison of the racemisation barriers among $1 \mathbf{c}$ and helicenes. Free energies of activation $(\mathrm{kcal} / \mathrm{mol})$ are shown in parentheses. $\mathbf{d}, \mathrm{NICS}(0)$ and $\mathrm{NICS}(1)$ values of the model structure (1c). Calculations were conducted at the GIAO-B3LYP/6-311+G(2d,p) level of theory. NICS(1) values are given in parentheses. Structural optimisation was conducted at the B3LYP/6-31G(d) level of theory. 
The nucleus-independent chemical shifts (NICS) were calculated to gain insight into the local aromaticity of each ring in compound $\mathbf{1}$ (Fig. 4d). According to the calculations, all the hexagonal rings (A, B, E, F, and G) show negative NICS(1) values, confirming their aromatic characters. In more detail, the A-B ring system, which would be expected to have naphthalene-like property, shows larger NICS(1) values than that of naphthalene ${ }^{50}(-10.7 \mathrm{ppm})$ especially for the B ring $(-6.98$ $\mathrm{ppm}$ ), suggesting the weaker local aromaticity of these rings. Similarly, the phenanthrene-like E-FG ring system shows larger NICS(1) values than that of unsubstituted phenanthrene. ${ }^{50}$ The weaker local aromaticity of these hexagonal rings can be attributed to the $\pi$-conjugations between $\mathrm{A}-\mathrm{B}$ and E-F-G ring systems through the embedded azulene structures. The pentagonal rings $(\mathrm{C}$ and $\mathrm{H})$ and the heptagonal ring (D) show a slightly negative NICS(1) value, indicating that these rings have significantly weaker aromaticity than that of the unsubstituted azulene ${ }^{50}(-7.82$ and $-17.8 \mathrm{ppm}$ for heptagon and pentagon, respectively), presumably due to the fused benzene rings (A, B, F, and G).

To investigate the optoelectronic and electrochemical properties of $\mathbf{1}$, UV-vis absorption spectroscopy, cyclic voltammetry (CV), and differential pulse voltammetry (DPV) were conducted. The UV-vis absorption spectra showed two weak bands at 660 and $600 \mathrm{~nm}$, and strong bands at 477 and $404 \mathrm{~nm}$ in the visible region (Fig. 5a). According to the TD-DFT calculation, the weak bands at 660 and $600 \mathrm{~nm}$ could be assigned as HOMO $\rightarrow$ LUMO $\left(\lambda_{\text {calc }}=635 \mathrm{~nm}, f=0.062\right)$ and HOMO-1 $\rightarrow$ LUMO transitions $\left(\lambda_{\text {calc }}=584, f=0.036\right)$, respectively (Fig. $\left.5 b\right)$. We assigned the strong band at 
$480 \mathrm{~nm}$ to the contributions of HOMO $\rightarrow$ LUMO $+1\left(\lambda_{\text {calc }}=548, f=0.247\right)$ and HOMO- $2 \rightarrow$ LUMO $\left(\lambda_{\text {calc }}=513, f=0.421\right)$ transitions. According to the CV analysis, compound 1a is stable to both electrochemical oxidation and reduction; it shows three reversible oxidation peaks at $0.72,0.83$ and 1.2 V, and two reduction peaks at -1.3 and $-1.7 \mathrm{~V}$ (vs. $\mathrm{Ag} / \mathrm{Ag}^{+}$) (Fig. 6a). In CV and DPV voltammograms, the first oxidation peak was split into two small peaks (P1 and P2). Variableconcentration DPV analysis of the soluble analogue $\mathbf{1 b}$ suggested that this splitting of the oxidation peaks resulted from the self-association characteristic of this compound (vide supra) (Fig. 6b). Since the first peak (P1) weakened with a decrease in the concentration, it is considered to be due to the oxidation of aggregated species. The second peak (P2), on the other hand, strengthened with a decrease in the concentration, indicating that it results from the oxidation of monomeric species. 
a

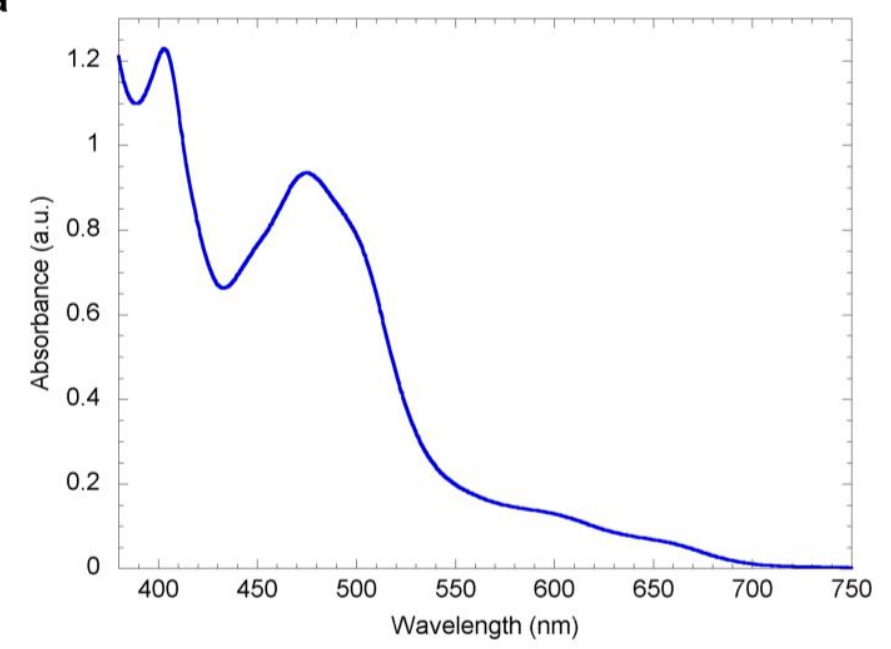

b

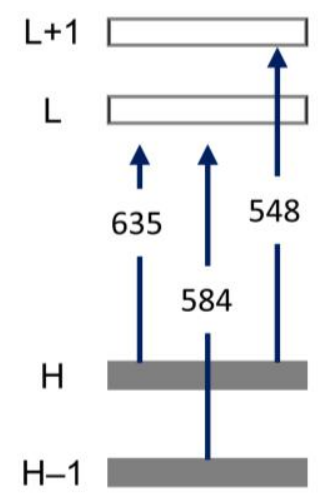

c
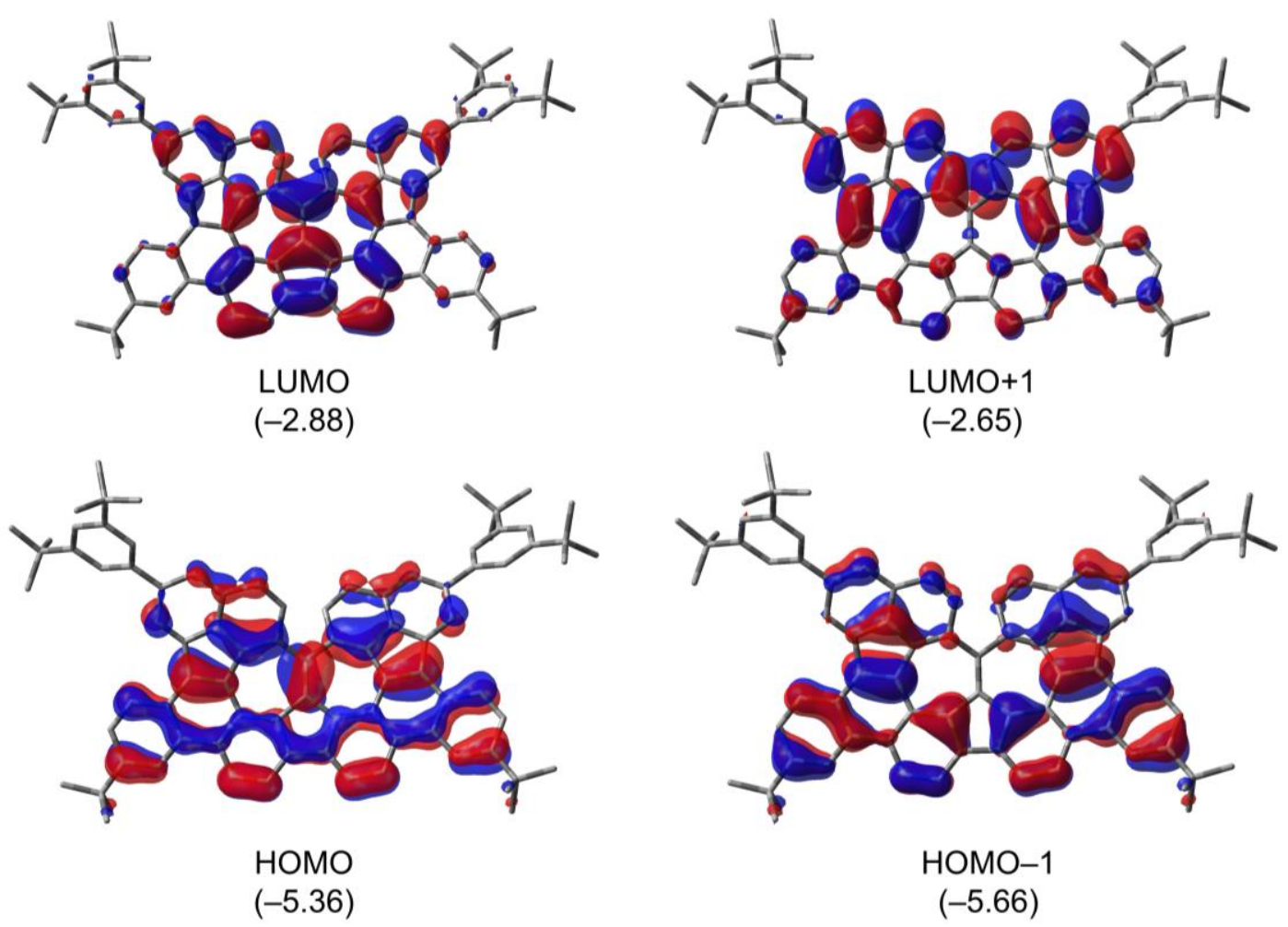

HOMO-1

$(-5.66)$

Fig. 5: Electronic properties of 1a. a, Experimental UV-vis absorption spectra of 1a $\left(1.0 \times 10^{-4} \mathrm{M}\right.$ in DCM). b, Major electronic transitions between frontier molecular orbitals of compound 1a calculated by the TD-DFT method at the B3LYP/6-311+G(d,p)-CPCM $\left(\mathrm{CHCl}_{3}\right)$ level of theory. Structural optimisation was conducted at the B3LYP/6-31G(d)-CPCM $\left(\mathrm{CHCl}_{3}\right)$ level of theory. Wave 
lengths (nm) are given. $\mathbf{c}$, Pictures of molecular orbitals of $\mathbf{1 a .}$

a

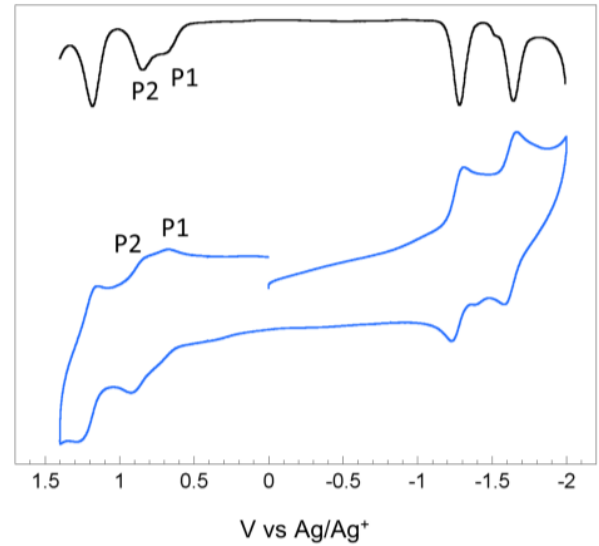

b

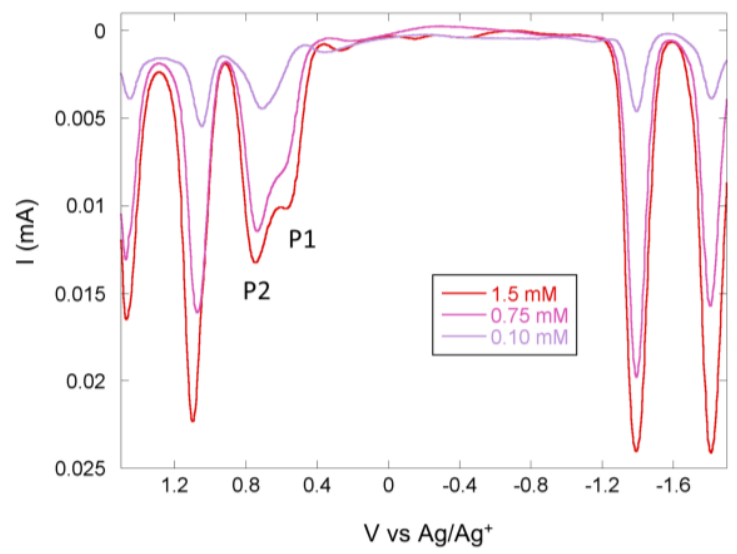

Fig. 6: Voltammograms of compounds 1a and 1b. a, Cyclic voltammogram (blue line) and

differential pulse voltammogram (black line) of $1 \mathbf{a}\left(1.0 \times 10^{-4} \mathrm{M}\right.$ in DCM). b, Variable-concentration differential pulse voltammogram of $\mathbf{1 b}\left(1.5 \times 10^{-3}, 7.5 \times 10^{-4}\right.$, and $1.9 \times 10^{-4} \mathrm{M}$ in DCM $)$.

In summary, a new methodology for the construction of a highly condensed azulene unit was developed to synthesise graphene nanostructure $\mathbf{1}$ featured by the embedded azulene cluster and a narrowed variant of the cove edge. The cove-like region of 1 comprising a 6-7-7-6 ring system was demonstrated to have stable chirality both experimentally and theoretically, in contrast with those of structures with normal cove edges. Single crystal X-ray analysis, variable-concentration NMR, and DPV analyses revealed the tendency of $\mathbf{1}$ to stack via $\pi-\pi$ interactions both in crystal form and in solutions. The new synthetic methodology and the information on the structural and electronic effect 
of the azulene cluster in graphene nanostructure provided herein would contribute to the future design and synthesis of various azulene-embedded nanocarbons.

\section{Methods}

Oxidative cyclisation of fluoranthene 2 c. A solution of $2 \mathbf{c}(16.2 \mathrm{mg}, 40.0 \mu \mathrm{mol})$ and DDQ (20.0 $\mathrm{mg}, 88.0 \mu \mathrm{mol})$ in dry $\operatorname{PrCN}(4.0 \mathrm{~mL})$ was irradiated with six $3.6 \mathrm{~W}$ blue LED strips for $60 \mathrm{~h}$ under argon. The reaction mixture was then diluted with $\mathrm{CHCl}_{3}$ and washed with saturated aqueous $\mathrm{NaHCO}_{3}$ followed by water. The organic phase was dried over $\mathrm{MgSO}_{4}$, filtered through a pad of silica gel, and then concentrated. The residue was purified by silica gel column chromatography (hexanes/EtOAc) to recover compound $\mathbf{3 c}$ (12.4 $\mathrm{mg}, 77 \%$ yield) as a purple solid.

Synthesis of 1a. To a stirred solution of $6(603 \mathrm{mg}, 0.541 \mathrm{mmol})$ and DDQ $(270 \mathrm{mg}, 1.19 \mathrm{mmol})$ in dry DCM (60 mL), TfOH $(0.47 \mathrm{~mL}, 5.4 \mathrm{mmol})$ was added. The mixture was stirred for $5 \mathrm{~min}$ and quenched with $\mathrm{Et}_{3} \mathrm{~N}$. The organic layer was washed with water, dried over $\mathrm{MgSO}_{4}$, and concentrated. Subsequently, the residue was dissolved in $\mathrm{CS}_{2}$, filtered through a pad of silica gel, and concentrated. After that, pentane was added to precipitate out the product, which was collected by filtration, washed several times with $\mathrm{MeOH}$ followed by pentane, and dried under reduced pressure to recover compound 1a (95.2 mg, 16\% yield) as a dark purple solid. 


\section{Data Availability}

All data generated and analysed during this study are included in this Article and its Supplementary Information. Crystallographic data for the structures reported in this Article have been deposited at the Cambridge Crystallographic Data Centre, under deposition number CCDC 1995289 (1a). Copies of the data can be obtained free of charge via www.ccdc.cam.ac.uk/data request/cif.

\section{References}

1. Yao, X. et al. Regioselective hydrogenation of a 60-carbon nanographene molecule toward a circumbiphenyl core. J. Am. Chem. Soc. 141, 4230-4234 (2019).

2. Chen, Q., Schollmeyer, D., Müllen, K. \& Narita, A. Synthesis of circumpyrene by alkyne benzannulation of brominated dibenzo[hi,st]ovalene. J. Am. Chem. Soc. 141, 19994-19999 (2019).

3. Zeng, W. et al. Superoctazethrene: An open-shell graphene-like molecule possessing large diradical character but still with reasonable stability. J. Am. Chem. Soc. 140, 14054-14058 (2018).

4. Ajayakumar, M. R. et al. Toward full zigzag-edged nanographenes: Peri-tetracene and its corresponding circumanthracene. J. Am. Chem. Soc. 140, 6240-6244 (2018).

5. Gregolińska, H. et al. Fully conjugated [4]chrysaorene. Redox-coupled anion binding in a 
tetraradicaloid macrocycle. J. Am. Chem. Soc. 140, 14474-14480 (2018).

6. Hitosugi, S. et al. Pentagon-embedded cycloarylenes with cylindrical shapes. Angew. Chem. Int. Ed. 56, 9106-9110 (2017).

7. Liu, J. et al. Unexpected Scholl reaction of 6,7,13,14-tetraarylbenzo[k]tetraphene: Selective formation of five-membered rings in polycyclic aromatic hydrocarbons. J. Am. Chem. Soc. 138, 2602-2608 (2016).

8. Cruz, C. M. et al. Enantiopure distorted ribbon-shaped nanographene combining two-photon absorption-based upconversion and circularly polarized luminescence. Chem. Sci. 9, 3917-3924 (2018).

9. Márquez, I. R. et al. Versatile synthesis and enlargement of functionalized distorted heptagoncontaining nanographenes. Chem. Sci. 8, 1068-1074 (2017).

10. Kawasumi, K., Zhang, Q., Segawa, Y., Scott, L. T. \& Itami, K. A grossly warped nanographene and the consequences of multiple odd-membered-ring defects. Nat. Chem. 5, 739-744 (2013).

11. Pun, S. H. et al. Synthesis, structures, and properties of heptabenzo[7]circulene and octabenzo[8]circulene. J. Am. Chem. Soc. 141, 9680-9686 (2019).

12. Michl, J. \& Thulstrup, E. W. Why is azulene blue and anthracene white? a simple MO picture.

Tetrahedron 32, 205-209 (1976).

13. Sun, Q. et al. On-surface synthesis of polyazulene with 2,6-connectivity. Chem. Commun. 55, 
13466-13469 (2019).

14. Ma, J. et al. Helical Nanographenes containing an azulene Unit: Synthesis, crystal structures, and properties. Angew. Chem. Int. Ed. 59, 5637-5642 (2020).

15. Yamaguchi, Y. et al. Terazulene isomers: Polarity change of OFETs through molecular orbital distribution contrast. J. Am. Chem. Soc. 138, 11335-11343 (2016).

16. Xin, H. et al. Biazulene diimides: A new building block for organic electronic materials. Chem. Sci. 7, 6701-6705 (2016).

17. Nishimura, H. et al. Hole-transporting materials with a two-dimensionally expanded $\pi$-system around an azulene core for efficient perovskite solar cells. J. Am. Chem. Soc. 137, 15656-15659 (2015).

18. Yazyev, O. V. \& Louie, S. G. Topological defects in graphene: Dislocations and grain boundaries. Phys. Rev. B 81, 195420 (2010).

19. Vicarelli, L., Heerema, S. J., Dekker, C. \& Zandbergen, H. W. Controlling defects in graphene for optimizing the electrical properties of graphene nanodevices. ACS Nano 9, 3428-3435 (2015).

20. Banhart, F., Kotakoski, J. \& Krasheninnikov, A. V. Structural defects in graphene. ACS Nano 5, 26-41 (2011).

21. Lu, J., Bao, Y., Su, C. L. \& Loh, K. P. Properties of strained structures and topological defects in graphene. ACS Nano 7, 8350-8357 (2013). 
22. Liu, J. et al. Toward cove-edged low band gap graphene nanoribbons. J. Am. Chem. Soc. 137,

6097-6103 (2015).

23. Nagarajan, K., Mallia, A. R., Muraleedharan, K. \& Hariharan, M. Enhanced intersystem crossing in core-twisted aromatics. Chem. Sci. 8, 1776-1782 (2017).

24. Sisto, T. J. et al. Long, Atomically precise donor-acceptor cove-edge nanoribbons as electron acceptors. J. Am. Chem. Soc. 139, 5648-5651 (2017).

25. Yano, Y. et al. Step-growth annulative $\pi$-extension polymerization for synthesis of cove-type graphene nanoribbons. J. Am. Chem. Soc. 142, 1686-1691 (2020).

26. Wu, J. et al. Cove-edged nanographenes with localized double bonds. Angew. Chemie Int. Ed. (2020). doi:10.1002/anie.202000326

27. Fan, Q. et al. Nanoribbons with nonalternant topology from fusion of polyazulene : Carbon allotropes beyond graphene. J. Am. Chem. Soc. 141, 17713-17720 (2019).

28. Wang, Y. et al. Template effect in the competition between Haeckelite and graphene growth on Ni(111): Quantum chemical molecular dynamics simulations. J. Am. Chem. Soc. 133, 18837$18842(2011)$.

29. Narita, A., Wang, X. Y., Feng, X. \& Müllen, K. New advances in nanographene chemistry.

Chem. Soc. Rev. 44, 6616-6643 (2015).

30. Segawa, Y., Ito, H. \& Itami, K. Structurally uniform and atomically precise carbon 
nanostructures. Nat. Rev. Mater. 1, 15002 (2016).

31. Yano, Y., Mitoma, N., Ito, H. \& Itami, K. A quest for structurally uniform graphene nanoribbons: synthesis, properties, and applications. J. Org. Chem. 85, 4-33 (2020).

32. Ziegler, K. \& Hafner, K. Eine rationelle Synthese des Azulens. Angew. Chem. 67, 301-301 (1955)

33. Lellek, V. \& Hansen, H. J. Surprising formation of highly substituted azulenes on thermolysis of 4,5,6,7,8-pentamethyl-2h-eyelohepta[b]furan-2-one and heptalene formation with the new azulenes. Helv. Chim. Acta 84, 1712-1736 (2001).

34. Carret, S. et al. Approach to the blues: A highly flexible route to the azulenes. Angew. Chem. Int. Ed. 44, 5130-5133 (2005).

35. Hieulle, J. et al. On-surface route for producing planar nanographenes with azulene moieties. Nano Lett. 18, 418-423 (2018).

36. Liu, J. et al. Open-shell nonbenzenoid nanographenes containing two pairs of pentagonal and heptagonal rings. J. Am. Chem. Soc. 141, 12011-12020 (2019).

37. Mastalerz, M., Yang, X. \& Rominger, F. Contorted polycyclic aromatic hydrocarbons with two embedded azulene units. Angew. Chemie Int. Ed. 58, 17577-17582 (2019).

38. Zhang, X. et al. Organic semiconductors dicyclohepta[ijkl,uvwx]rubicene with two pentagons and two heptagons as a stable and planar non-benzenoid nanographene. Angew. Chem. Int. Ed. 59, 
$3529-3533$ (2019).

39. Ogawa, N., Yamaoka, Y., Yamada, K. \& Takasu, K. Synthesis of $\pi$-extended fluoranthenes via a KHMDS-promoted anionic-radical reaction cascade. Org. Lett. 19, 3327-3330 (2017).

40. Mallory, F. B. \& Mallory, C. W. Photocyclization of stilbenes and related molecules. Org. React. 30, 1-449 (1984).

41. Villedieu-Percheron, E. et al. $6 \pi / 10 \pi$-Electrocyclization of ketene-iminium salts for the synthesis of substituted naphthylamines. Tetrahedron Lett. 55, 2446-2449 (2014).

42. Gobbi, L. et al. Photoswitchable tetraethynylethene-dihydroazulene chromophores. Helv. Chim. Acta 84, 743-777 (2001).

43. Miyaura, N. \& Suzuki, A. Palladium-catalyzed cross-coupling reactions of organoboron compounds. Chem. Rev. 95, 2457-2483 (1995).

44. Sheldrick, G. M. A short history of SHELX. Acta Crystallogr. Sect. A Found. Crystallogr. 64, $112-122$ (2008).

45. Dittrich, B. et al. Azulene revisited: solid-state structure, invariom modeling and lattice-energy minimization of a classical example of disorder. Acta Cryst. B 74, 416-426 (2018).

46. Nakamura, K., Okubo, H. \& Yamaguchi, M. Synthesis and self-aggregation of cyclic alkynes containing helicene. Org. Lett. 3, 1097-1099 (2001).

47. Tobe, Y. et al. m-Diethynylbenzene macrocycles: Syntheses and self-association behavior in 
solution. J. Am. Chem. Soc. 124, 5350-5364 (2002).

48. Barroso, J. et al. Revisiting the racemization mechanism of helicenes. Chem. Commun. 54, 188$191(2017)$

49. Martin, R. H. \& Marchant, M. J. Thermal racemisation of hepta-, octa-, and nonahelicene. Kinetic results, reaction path and experimental proofs that the racemisation of hexa- and heptahelicene does not involve an intramolecular double diels-alder reaction. Tetrahedron 30, 347-349 (1974).

50. Chen, Z., Wannere, C. S., Corminboeuf, C., Puchta, R. \& von Ragué Schleyer, P. Nucleusindependent chemical shifts (NICS) as an aromaticity criterion. Chem. Rev. 105, 3842-3888 (2005). 


\section{Acknowledgements}

We acknowledge financial support provided by JSPS KAKENHI to K.T. (Grant Nos. JP19K22185, JP19H03350, and JP18H04406), JSPS for a research fellowship for young scientists to N.O.

(JP19J14061), a Platform for Drug Design, Discovery and Development grant from MEXT to K.T., and the Sasakawa Scientific Research Grant from The Japan Science Society to N.O. We thank Prof. Takashi Kubo (Graduate School of Science, Osaka University) for CV and DPV analyses, Dr. Yoshiaki Yano (Graduate School of Pharmaceutical Sciences, Kyoto University) for CD analyses, and Prof. Toru Nakatsu (Graduate School of Pharmaceutical Sciences, Kyoto University) and Dr. Hiroyasu Sato (Rigaku Corporation) for X-ray crystallographic analyses. In this research work we used the supercomputer of ACCMS, Kyoto University.

\section{Contributions}

N.O. and K.T. developed the concept and conceived the experiments. N.O. performed the experiments. Y.Y., H.T., and K.Y. analysed the data. N.O. prepared the manuscript with assistance from K.T.

\section{Ethics declarations}

\section{Competing interests}


The authors declare no competing interests.

\section{Supplementary information}

Supplementary information and chemical compound information are available in the online version of the paper. Reprints and permissions information is available online at www.nature.com/reprints.

Correspondence and requests for materials should be addressed to K.T. 
Fig. 1: Azulene-embedded graphenes and graphene nanostructures. a, One example of a graphene nanoribbon containing clusters of azulene units. b, Structures of "normal" cove edge. c, "Narrowed" variant of cove edge. $\mathbf{d}$, Structural design and synthetic strategy of nanographene $\mathbf{1}$

Fig. 2: Synthetic protocol for the azulene units. a, Conversion of dibenzofluoranthene 2 into azulene 3 via visible-light-promoted oxidative cyclisation. b, Synthesis of AGN 1a. Reaction conditions: (a) $\mathrm{Tf}_{2} \mathrm{O}$, DIPEA, DCM, $25^{\circ} \mathrm{C}$ (b) $\mathrm{CH}_{2}=\mathrm{C}(\mathrm{Bpin})_{2}, \mathrm{~K}_{3} \mathrm{PO}_{4}, \mathrm{Pd}\left(\mathrm{PPh}_{3}\right)_{4}$, dioxane, $100{ }^{\circ} \mathrm{C}$,

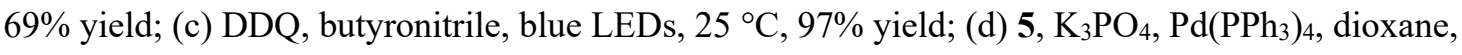
$100{ }^{\circ} \mathrm{C}, 46 \%$ yield; (e) DDQ, TfOH, DCM, $25^{\circ} \mathrm{C}, 5 \mathrm{~min}, 16 \%$ yield. $\mathrm{Tf}=$ trifluoromethanesulfonyl; DIPEA = diisopropylethylamine; $\mathrm{DCM}=$ dichloromethane; pin = pinacolate; $\mathrm{DDQ}=2,3$-dichloro5,6-dicyano-p-benzoquinone; LED = light emitting diode.

Fig. 3: Crystal structure of 1a. Hydrogen atoms and solvents are omitted for clarity. a, Top view (thermal ellipsoids are shown at 50\% probability). b, Bond length of compound 1a (left) and azulene (right). c, Top view and, $\mathbf{d}$, side view of the packing structure in the crystal.

Fig. 4: Chirality and local aromaticity of AGN 1. a, CD spectra of $1 \mathbf{b}\left(5.0 \times 10^{-5} \mathrm{M}\right.$ in hexane). $\mathbf{b}$, 
Process for the racemisation of the model compound 1c. Relative Gibbs free energies ( $\mathrm{kcal} / \mathrm{mol})$ are given in parentheses. Calculation was carried out at the B3LYP/6-31G(d,p) level of theory. c, Comparison of the racemisation barriers among $1 \mathbf{c}$ and helicenes. Free energies of activation $(\mathrm{kcal} / \mathrm{mol})$ are shown in parentheses. d, NICS(0) and NICS(1) values of the model structure (1c). Calculations were conducted at the GIAO-B3LYP/6-311+G(2d,p) level of theory. NICS(1) values are given in parentheses. Structural optimisation was conducted at the B3LYP/6-31G(d) level of theory.

Fig. 5: Electronic properties of 1a. a, Experimental UV-vis absorption spectra of 1a $\left(1.0 \times 10^{-4} \mathrm{M}\right.$ in DCM). b, Major electronic transitions between frontier molecular orbitals of compound 1a calculated by the TD-DFT method at the B3LYP/6-311+G(d,p)-CPCM $\left(\mathrm{CHCl}_{3}\right)$ level of theory. Structural optimisation was conducted at the B3LYP/6-31G(d) level of theory. $\mathbf{c}$, Pictures of molecular orbitals of $\mathbf{1 a}$.

Fig. 6: Voltammograms of compounds 1a and 1b. a, Cyclic voltammogram (blue line) and differential pulse voltammogram (black line) of $\mathbf{1 a}\left(1.0 \times 10^{-4} \mathrm{M}\right.$ in DCM). b, Variable-concentration differential pulse voltammogram of $\mathbf{1 b}\left(1.5 \times 10^{-3}, 7.5 \times 10^{-4}\right.$, and $1.9 \times 10^{-4} \mathrm{M}$ in DCM $)$. 